\title{
CUSTO DO TRATAMENTO CONVENCIONAL E COM BRENTUXIMAB NOS PACIENTES COM LINFOMA DE HODGKIN
}

\author{
A. H. B. de ARAUJO ${ }^{1}$, R. V. C. $\operatorname{SANTOS}^{2}$ e M. J. B. de M. RÊGO ${ }^{1,2}$ \\ ${ }^{1}$ Universidade Federal de Pernambuco, Centro de Ciências Sociais Aplicadas \\ ${ }^{2}$ Universidade Federal de Pernambuco, Centro de Biociências, Núcleo de Pesquisa em \\ Inovação Terapêutica
}

E-mail para contato: adrianaaheleny@gmail.com

\begin{abstract}
RESUMO - O Linfoma de Hodgkin é um câncer que na maioria das vezes tem excelente prognóstico com tratamento convencional. Todavia, podem acontecer casos de recidiva ou falência no tratamento. Para esses casos surgiu no mercado o antineoplásico Brentuximab. Este trabalho é um estudo descritivo do tipo revisão da literatura e tem como objetivo comparar os custos com tratamento convencional e os custos com o Brentuximab, relacionando com a efetividade da medicação. Foram utilizados os termos Doença de Hodgkin; Inovação Terapêutica; Tratamento; Custo-efetividade nas bases de dados Scielo, Medline, Pubmed, A Cancer Journal for Clinicians, selecionando-se 4 artigos; além de outras fontes de pesquisa. O custo médio para o tratamento convencional em estágio limitado desfavorável foi de $R \$ 8.860,06$; enquanto que no estágio avançado o valor ficou em $R \$ 11.655,09$. O tratamento com o Brentuximab teve um custo estimado superior a $R \$ 500.000,00$ por paciente. Estudos mostram que cerca de um terço dos pacientes utilizando Brentuximab obtiveram remissão completa ou aumento significativo da sobrevida livre de progressão, quando comparados com placebo ou tratamentos clássicos. Todavia, seus efeitos adversos podem ser muito graves, como no caso da hepatoxicidade e toxicidade pulmonar. Diante do contexto demonstrado do alto custo do tratamento com Brentuximab e seus beneficios/efeitos colaterais, torna-se imprescindivel a realização de mais estudos relacionados.
\end{abstract}

Palavras-chave: Doença de Hodgkin; Inovação Terapêutica; Tratamento; Custoefetividade

ABSTRACT - Hodgkin's lymphoma is a cancer that in most cases has an excellent prognosis with conventional treatment. However, cases of recurrence or failure in treatment may occur. For these cases the antineoplastic Brentuximab appeared in the market. This study is a descriptive study of the type literature review and aims to compare costs with conventional treatment and costs with Brentuximab, relating 
to the effectiveness of the medication. The terms Hodgkin's disease were used; Therapeutic Innovation; Treatment; Cost-effectiveness in the databases Scielo, Medline, Pubmed, The Cancer Journal for Clinicians, selecting 4 articles; as well as other sources of research. The average cost for conventional treatment at an unfavorable limited stage was $R \$ 8,860.06$; while in the advanced stage the value was $R \$ 11,655.09$. Treatment with Brentuximab had an estimated cost of more than $R \$ 500,000$ per patient. Studies show that about one-third of patients using Brentuximab achieved complete remission or significantly increased progressionfree survival when compared with placebo or classical treatments. However, its adverse effects can be very serious, as in the case of hepatic toxicity and pulmonary toxicity. In view of the demonstrated context of the high cost of treatment with Brentuximab and its benefits / side effects, it is essential to carry out more related studies.

Keywords: Hodgkin's disease; Therapeutic innovation; Treatment; Costeffectiveness

\section{INTRODUÇÃO}

O Linfoma de Hodgkin (LH) é um câncer que se origina nos linfonodos, após proliferação descontrolada das células de defesa do organismo. Geralmente tem excelente prognóstico após quimioterapia (QT) e/ou radioterapia (RT). Todavia, cerca de $35 \%$ dos pacientes apresenta falência no tratamento. Das inovações terapêuticas, surgiu no mercado o Brentuximab, antineoplásico de alto custo que promete um aumento da sobrevida nos casos mais difíceis. A medicação obteve registro na Agência Nacional de Vigilância Sanitária (ANVISA) em setembro de 2014, mas ainda não está presente na rede do Sistema Único de Saúde no Brasil e aguarda avaliação da Comissão Nacional de Incorporação de Tecnologias - CONITEC (ABRALE, 2016; PORTO, C.C., 2011; ARANTES, A.M., 2011; FERNANDES, B.V, 2013; ONCONEWS, 2015).

Até o momento não foram encontrados estudos científicos no Brasil direcionados ao uso e custo-efetividade do medicamento em questão. Entretanto, as judicializações para obtenção e tratamento com o Brentuximab vêm crescendo substancialmente no país, podendo levar a um impacto econômico importante. $O$ objetivo dessa revisão foi comparar os custos relacionados ao tratamento convencional e os custos com o Brentuximab, bem como verificar estudos que evidenciem uma possível efetividade da medicação (O Estado de S. Paulo, 2016; TRF-4, 2017).

\section{MATERIAIS E MÉTODOS}

Trata-se de um estudo descritivo, no qual os descritores utilizados foram Doença de Hodgkin; Inovação terapêutica; Tratamento; Custo-efetividade. Foram utilizadas as seguintes fontes para pesquisa: bases de dados (Scielo, Medline, Pubmed, A Cancer Journal for Clinicians); Google acadêmico; sites oficiais relacionados ao câncer; revistas e jornais on-line; livros; monografias e dissertações. 


\section{RESULTADOS E DISCUSSÃO}

De todos os artigos científicos encontrados nas bases de dados, foram selecionados 4 contendo o que buscávamos para o trabalho. A QT é a escolha para tratamento clássico do LH. O esquema quimioterápico de primeira linha para a doença em estágio inicial é o ABVD (doxorrubicina + bleomicina + vimblastina + dacarbazina), além da RT. A taxa de sobrevida dos pacientes após tratamento inicial é de, no mínimo 5 anos e chega a aproximadamente $90 \%$. Entretanto, cerca de $10 \%$ dos pacientes com LH localizado e $25 \%$ com doença avançada, apresentam uma resistência ao tratamento clássico com falência de resposta deste. Os efeitos colaterais mais comuns da QT são enjoo, xerostomia, diarreia, entre outros. Porém, podem surgir outros importantes, como a inflamação dos pulmões quando utilizada a medicação Bleomicina (OLIVEIRA, C.I.A.L, 2016; BITTENCOURT, R.I; et al, 2010; AMERICAN CANCER SOCIETY, 2017; ABRALE, 2016).

A fim de aumentar a sobrevida dos pacientes com refratariedade secundária, surgiram alternativas terapêuticas, como o Brentuximab, uma medicação produzida em laboratório que associa um anticorpo anti CD-30 e o fármaco monometil auristatina $\mathrm{E}$. Este medicamento ainda não foi avaliado pela Comissão Nacional de Incorporação de Tecnologias - CONITEC, mas possui registro na ANVISA desde setembro de 2014 (ONCOGUIA, 2015; SANTOS, F. M.; et al, 2008; MOSKOWITZ, C.H.; et al, 2015; EMA, 2017; CATES, 2016).

Segundo a Associação Brasileira de Linfoma e Leucemia, no ano de 2016, o custo médio para o tratamento do LH em estágio limitado desfavorável (III e IV), sendo QT (ABVD) em 4 ciclos + RT, correspondeu a R\$ 8.860,06; enquanto que o tratamento no estágio avançado (V), com QT (ABVD) em 6 ciclos o valor foi de R\$ 11.655,09 (ABRALE, 2016).

Por outro lado, o tratamento com o Brentuximab pode ter um custo superior a $\mathrm{R} \$$ $500.000,00$ por paciente, se levado em conta a dose recomendada pelo fabricante que é de $1,8 \mathrm{mg} / \mathrm{kg}$, devendo ser administrada a cada 3 semanas, numa quantidade mínima de 8 doses com no máximo 16 ciclos, conforme mostra a tabela 1. Esses valores são da Câmara de Regulação do Mercado de Preços que está inserida na ANVISA, considerando o ICMS de 18\% e 0\% (imposto desonerado), no ano de 2016 (CATES, 2016).

Tabela 1 - Preço estimado do tratamento com Brentuximab por ciclo (Centro Colaborador do SUS, 2016).

\begin{tabular}{|c|c|c|c|}
\hline ICMS & $\begin{array}{c}\text { Dose por ciclo para } \\
\text { paciente com 70 kg }\end{array}$ & Apresentação & Valor do ciclo \\
\hline $0 \%$ & 126 miligramas & $\begin{array}{c}50 \text { miligramas pó } \\
\text { liofilizado injetável caixa } \\
\text { com um frasco }\end{array}$ & R $31.115,58$ \\
\hline $18 \%$ & 126 miligramas & $\begin{array}{c}50 \text { miligramas pó } \\
\text { liofilizado injetável caixa } \\
\text { com um frasco }\end{array}$ & R $38.991,27$ \\
\hline
\end{tabular}

Em um estudo realizado em 25 centros situados nos Estados Unidos, Canadá e Europa, foram selecionados 102 pacientes com histórico de LH células CD-30 positivas, anteriormente tratados com QT de alta dose e TACT, apresentando recidiva ou falência do tratamento. Nesse estudo, $34 \%$ dos pacientes obtiveram remissão completa da doença 
com Brentuximab. Além de reduções dos tumores em 94\% dos casos e a doença geral com taxa de controle de $96 \%$. Após 1,5 anos de observação, 31 dos 102 pacientes estavam vivos e sem histórico de progressão do LH (YOUNES, A.; et al, 2012)

Em outro estudo multicêntrico de ensaio clínico randomizado, duplo cego, controlado com placebo, realizado na América do Norte e Europa com recrutamento de 329 pacientes que já haviam realizado TACT, também foi constatado um resultado positivo. A sobrevida livre de progressão da doença foi significativamente maior nos pacientes tratados com Brentuximab (42,9 meses) quando comparados com os que utilizaram o placebo $(24,1$ meses). Entretanto, em 02 anos a sobrevida foi de $63 \%$ no grupo do Brentuximab e $51 \%$ no grupo placebo, levando a constatar que a sobrevida global e sobrevida livre da progressão da doença não tiveram diferenças significativas nesse período. Além disso, foram observados alguns efeitos adversos importante, como neuropatia sensorial periférica e neutropenia (MOSKOWITZ, C.H.; et al, 2015; CATES, 2016).

De acordo com a Agência Europeia de Medicamentos (EMA), são vários os efeitos adversos graves e potencialmente fatais que podem estar associados ao uso do Bretuximab, como a toxicidade pulmonar; infecções graves e oportunistas; neuropatia periférica, sendo reversível na maioria dos casos; Síndrome de Stevens -Johnson e Necrólise Epidérmica Tóxica; hepatoxicidade; comprometimento renal; hiperglicemia, entre outros. Diante do quadro atual, a EMA aprovou condicionalmente a medicação, afirmando haver, até o momento, exposição de maiores benefícios quando comparados aos riscos, porém, com ressalva da necessidade de ampliar as pesquisas a fím de confirmar os efeitos do medicamento a longo prazo e comparação com outros tratamentos para o LH (EMA, 2017; CATES, 2016).

\section{CONCLUSÃO}

Conclui-se que Brentuximab possui vantagens importantes relacionadas ao seu uso, porém, torna-se imprescindível pesquisas mais aprofundadas e a análise criteriosa do custo benefício do tratamento com o mesmo, tendo em vista o alto custo da medicação, especialmente quando comparado com o tratamento convencional, o tempo de sobrevida após seu uso, bem como os efeitos adversos associados.

\section{REFERÊNCIAS}

ASSOCIAÇÃO BRASILEIRA DE LINFOMA E LEUCEMIA - ABRALE. Linfoma de Hodgkin - LH. Abril, 2016. Disponível em: http://www.abrale.org.br/lh/o-que-e. Acesso em 20 de agosto de 2017.

ARANTES, A.M.; et al. Transplante autólogo de células-tronco em linfoma de Hodgkin: análise de uma opção terapêutica. Hospital Araújo Jorge - HAJ. Goiânia, 2011. Disponível em: http://www.scielo.br/pdf/eins/v9n2/pt 1679-4508-eins-9-2-0124.pdf. Acesso em 05 de setembro de 2017.

BITTENCOURT, R.I. et al. Transplante de células-tronco hematopoéticas em linfoma Hodgkin. Rev. Bras. Hematol. Hemoter. vol.32 supl.1 São Paulo May 2010 Epub Apr 02, 
2010. Disponível em: http://www.scielo.br/scielo.php?script=sci_arttext\&pid=S151684842010000700015. Acesso em 03 de outubro de 2017.

CENTRO COLABORADOR DO SUS - CATES. Brentuximabe para o tratamento do Linfoma de Hodgkin. Universidade Federal de Minas Gerais. Belo Horizonte, 2016. Disponível em: http://www.ccates.org.br/content/ pdf/PUB 1492436676.pdf. Acesso em 08 de setembro de 2017.

FERNANDES, B.V. Frequência de segundas neoplasias após o tratamento para linfoma de Hodgkin: um estudo descritivo e revisão de literatura. MONOGRAFIA. Salvador, 2013.

HARTT, V. Inovação em linfoma de Hodgkin não é realidade no SUS. ONCONEWS, 2015 Disponível em: http:/www.onconews.com.br/site/noticias/noticias/ultimas/1196inova $\% \mathrm{C} 3 \% \mathrm{~A} 7 \% \mathrm{C} 3 \% \mathrm{~A} 30$-em-linfoma-de-hodgkin-n\%C3\%A3o-\%C3\%A9-realidade-nosus.html. Acesso em 05 de setembro de 2017.

MOSKOWITZ, C.H.; et al. Brentuximab vedotin as consolidation therapy after autologous stem-cell transplantation in patients with Hodgkin's lymphoma at risk of relapse or progression (AETHERA): a randomised, double-blind, placebo-controlled, phase 3 trial. $\quad$ Lancet, 2015. Disponível em: https://www.ncbi.nlm.nih.gov/pubmed/25796459. Acesso em 08 de setembro de 2017.

O Estado de S.Paulo. 'Eu não teria condições de pagar por tratamento', diz músico. 2016. Disponível em: http://saude.estadao.com.br/noticias/geral,eu-nao-teria-condicoes-de-pagarpor-tratamento-diz-musico,10000078850. Acesso em 11 de setembro de 2017.

OLIVEIRA, C.I.A.L. Estratégias terapêuticas no Linfoma de Hodgkin no séc. XXI. Mestrado Integrado em Medicina. Porto, 2016. Disponível em: https://repositorioaberto.up.pt/bitstream/10216/86476/2/165930.pdf. Acesso em 14 de setembro de 2017.

PORTO, C.C. Vademecum de clínica médica. Rio de Janeiro: Guanabara Koogan, 2011.

YOUNES, A.; et al. Results of a Pivotal Phase II Study of Brentuximab Vedotin for Patients With Relapsed or Refractory Hodgkin's Lymphoma. J Clin Oncol. vol.30 n.18 Junho, 2012. Disponível em: https://www.ncbi.nlm.nih.gov/pubmed/22454421. Acesso em 11 de setembro de 2017.

\section{AGRADECIMENTOS}

Os autores expressam seus agradecimentos à Universidade Federal de Pernambuco, Pró-Reitoria de Planejamento, Orçamento e Finanças e ao Ministério da Saúde pela concessão da bolsa, ajuda de custo e contribuição para desenvolvimento da pesquisa. 